\title{
LINEAR FILTERING WITH FRACTIONAL BROWNIAN MOTION IN THE SIGNAL AND OBSERVATION PROCESSES
}

\author{
M.L. KLEPTSYNA \\ Russian Academy of Sciences \\ Institute of Information Transmission Problems \\ Moscow 101447, Russia \\ marina@sci.lpi.ac.ru \\ P.E. KLOEDEN \\ Johan Wolfgang Goethe Universität \\ Fachbereich Mathematik \\ D-60054 Frankfurt am Main Germany \\ kloeden@math.uni-frankfurt.de \\ V.V. ANH \\ Queensland University of Technology \\ School of Mathematical Sciences \\ Brisbane 4001, Australia \\ v.anh@fsc.qut.edu.au
}

(Received December, 1997; Revised July, 1998)

Integral equations for the mean-square estimate are obtained for the linear filtering problem, in which the noise generating the signal is a fractional Brownian motion with Hurst index $h \in(3 / 4,1)$ and the noise in the observation process includes a fractional Brownian motion as well as a Wiener process.

Key words: Linear Filtering, Fractional Brownian Motion, LongRange Dependence, Optimal Mean-Square Filter.

AMS subject classifications: $93 \mathrm{E} 11,60 \mathrm{G} 20,60 \mathrm{G} 35$.

\section{Introduction}

We consider the linear problem with the signal $\theta_{t}$ and the observation $\xi_{t}$ defined by the linear equations

$$
\theta_{t}=\int_{0}^{t} a(s) \theta_{s} d s+B_{t}^{h}, \xi_{t}=\int_{0}^{t} A(s) \theta_{s} d s+W_{t}+B_{t}^{h}
$$


where the noise generating the signal is a fractional Brownian motion (fBm) $B_{t}^{h}$ with Hurst index $h \in(3 / 4,1)$ and the noise disturbing the observation of the signal consists of both a standard Wiener process $W_{t}$ and the fractional Brownian motion $B_{t}^{h}$. The coefficients $a(t)$ and $A(t)$ are bounded measurable functions and the noise processes $B_{t}^{h}$ and $W_{t}$ are independent.

Fractional Brownian motion $B_{t}^{h}$ with Hurst index $h \in(1 / 2,1)$ is often used to model the long-range dependence in random data commonly encountered in many financial and environmental applications $[7,9]$. It is a zero mean Gaussian process having the correlation function

$$
\Gamma^{h}(t, s)=\frac{1}{2}\left(t^{2 h}+s^{2 h}-|t-s|^{2 h}\right), 1 / 2<h<1 .
$$

It is known that $B_{t}^{h}$ is not a semimartingale (see e.g. $[4,6]$ ), so neither is the signal process $\theta_{t}$ nor the observation process $\xi_{t}$, and the martingale approach to filtering expounded in [6] is not applicable here. In particular, as shown in [8], we cannot uniquely determine an innovation process corresponding to $\xi_{t}$. Nevertheless, we can derive an explicit expression for the conditional expectation of the signal

$$
\widehat{\theta}_{t} \triangleq \mathbb{E}\left(\theta_{t} \mid \xi_{s}, 0 \leq s \leq t\right)
$$

using a theorem on normal correlation in [5] provided we restrict the Hurst index $h$ to the interval $(3 / 4,1)$. We formulate this results as a theorem in the next section and present its proof in Section 3. Finally, a simple example is provided in Section 4 to illustrate the result.

\section{The Optimal Filter}

Let $\mathcal{F}_{t}^{\xi}$ be the $\sigma$-algebra $\sigma\left(\xi_{s}, 0 \leq s \leq t\right)$ and note that $\widehat{\theta}_{t}=\mathbb{E}\left(\theta_{t} \mid \mathcal{F}_{t}^{\xi}\right)$. Define

$$
K(t, s) \triangleq \mathbb{E}\left(\theta_{t} \theta_{s}\right), \quad \tilde{K}(t, s) \triangleq \mathbb{E}\left(\theta_{t} B_{s}^{h}\right) .
$$

Then it follows directly from the first equation of (1) that $K(t, s)$ and $\widetilde{K}(t, s)$ satisfy the system of integral equations

$$
\begin{aligned}
& K(t, s)=\int_{0}^{s} a(l) K(t, l) d l+\widetilde{K}(t, s), \\
& \widetilde{K}(t, s)=\int_{0}^{t} a(l) \widetilde{K}(l, s) d s+\Gamma^{h}(t, s) .
\end{aligned}
$$

With these we can obtain an explicit closed-form representation of the optimal meansquare filter for system (1).

Theorem 2.1: There exists a unique deterministic function $\Phi \in L^{2}\left([0, T]^{2}, \mathbb{R}\right)$ satisfying

$$
\Phi(t, s)=-\int_{0}^{s} \Phi(t, \tau)\left[h(2 h-1)|s-\tau|^{2 h-2}\right.
$$




$$
\begin{aligned}
+A(s) A(\tau) K( & \left.\tau, s)+A(\tau) \frac{\partial \widetilde{K}}{\partial s}(\tau, s)+A(s) \frac{\partial \widetilde{K}}{\partial \tau}(s, \tau)\right] d \tau \\
& +A(s) K(t, s)+\frac{\partial \widetilde{K}}{\partial s}(t, s)
\end{aligned}
$$

such that the optimal mean-square filtering estimate $\hat{\theta}_{t}$ of the linear system (1) satisfies

$$
\widehat{\theta}_{t}=\int_{0}^{t} \Phi(t, s) d \xi_{s}
$$

for $t \in[0, T]$, where the integral is understood in the mean-square sense.

It follows from the proof of Theorem 1 that system (5) has a solution. This solution is in fact unique.

Theorem 2.2: The system of integral equations (3)-(5) has a unique solution.

\section{Proof of Theorem 1}

We note that the joint distribution of $\left(\xi_{s}, \theta_{t}\right)$ for all $0 \leq s, t \leq T$ is Gaussian, so Theorem 13.1 of [5] on normal correlation holds here. Let $0=t_{0}^{(n)}<t_{1}^{(n)}<\ldots<$ $t_{2}^{(n)}=t$ be the dyadic partition of $[0, t]$, that is, with $t_{j}^{(n)}=\frac{j}{2^{n}} t$ for $j=0,1, \ldots, 2^{n}$,

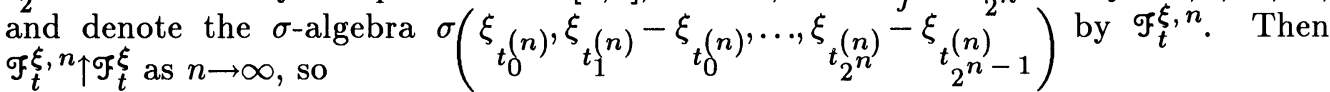

$$
\mathbb{E}\left(\theta_{t} \mid \mathcal{F}_{t}^{\xi, n}\right) \rightarrow \mathbb{E}\left(\theta_{t} \mid \mathcal{F}_{t}^{\xi}\right), n \rightarrow \infty
$$

for all $t \in[0, T]$. Furthermore,

$$
\lim _{n \rightarrow \infty} \mathbb{E}\left[\mathbb{E}\left(\theta_{t} \mid \mathcal{F}_{t}^{\xi, n}\right)-\mathbb{E}\left(\theta_{t} \mid \mathcal{F}_{t}^{\xi}\right)\right]^{2}=0
$$

for all $t \in[0, T]$. Hence using Theorem 13.1 of [5] we obtain

$$
\mathbb{E}\left(\theta_{t} \mid \mathcal{F}_{t}^{\xi, n}\right)=\mathbb{E}\left(\theta_{t} \mid \mathcal{F}_{t}^{\xi}\right)+\sum_{j=1}^{2 n-1} \Phi_{n}\left(t, t_{j}^{(n)}\right)\left(\xi_{t_{j+1}^{(n)}}-\xi_{t_{j}^{(n)}}\right)
$$

for all $t \in[0, T]$, where $\Phi_{n}:[0, T]^{2} \rightarrow \mathbb{R}$ is a deterministic function. Denote $\Phi(t, s)=$ $\Phi_{n}\left(t, t_{j}^{(n)}\right)$ for $t_{j}^{(n)} \leq s<t_{j+1}^{(n)}$. Then we can rewrite (8) as

$$
\mathbb{E}\left(\theta_{t} \mid \mathcal{F}_{t}^{\xi, n}\right)=\int_{0}^{t} \Phi_{n}(t, s) d \xi_{s}
$$

But the processes $W_{t}$ and $\left(B_{t}^{h}, \theta_{t}\right)$ are independent, so

$$
\mathbb{E}\left[\mathbb{E}\left(\theta_{t} \mid \mathcal{F}_{t}^{\xi, n}\right)-\mathbb{E}\left(\theta_{t} \mid \mathcal{F}_{t}^{x, m}\right)\right]^{2}=\int_{0}^{t}\left|\Phi_{n}(t, s)-\Phi_{m}(t, s)\right|^{2} d s
$$




$$
+\mathbb{E}\left\{\int_{0}^{t} \Phi_{n}(t, s)\left(d B_{s}^{h}+A(s) \theta_{s} d s\right)-\int_{0}^{t} \Phi_{m}(t, s)\left(d B_{s}^{h}+A(s) \theta_{s} d s\right)\right\}^{2} .
$$

(The integration of a deterministic function with respect to an $\mathrm{fBm}$ here is understood in the mean-square sense, cf. [4]). Applying (7) we obtain that

$$
\lim _{n, m \rightarrow \infty} \int_{0}^{t}\left|\Phi_{n}(t, s)-\Phi_{m}(t, s)\right|^{2} d s=0
$$

so the sequence $\left\{\Phi_{n}\right\}$ is a Cauchy sequence in $L^{2}[0, t]$. Hence there exist a function $\Phi \in L^{2}[0, t]$ such that

$$
\lim _{n \rightarrow \infty} \int_{0}^{t}\left|\Phi_{n}(t, s)-\Phi(t, s)\right|^{2} d s=0
$$

It then follows from [1] that

and

$$
\lim _{n \rightarrow \infty} \mathbb{E}\left(\left|\int_{0}^{t} \Phi_{n}(t, s) d B_{s}^{h}-\int_{0}^{t} \Phi(t, s) d B_{2}^{h}\right|^{2}\right)=0
$$

$$
\lim _{n \rightarrow \infty} \mathbb{E}\left(\left|\int_{0}^{t} \Phi_{n}(t, s) A(s) \theta_{s} d s-\int_{0}^{t} \Phi(t, s) A(s) \theta_{s} d s\right|^{2}\right)=0
$$

so we obtain

$$
\widehat{\theta}_{t}=\int_{0}^{t} \Phi(t, s) d \xi_{s}
$$

We shall now show that $\Phi$ satisfies equation (5). Let $f:[0, T]^{2} \rightarrow \mathbb{R}$ be a bounded and jointly measurable function, so the integral

$$
I_{t}:=\int_{0}^{t} f(t, s) d \xi_{s}
$$

is well-defined and the process $I_{t}$ is $\mathcal{F}_{t}^{\xi}$-measurable with $\mathbb{E}\left(I_{t}^{2}\right)<\infty$ and $\mathbb{E}\left(\left(\theta_{t}-\right.\right.$ $\left.\left.\widehat{\theta}_{t}\right) I_{t}\right)=0$. Consequently

$$
\begin{gathered}
\mathbb{E}\left(\theta_{t} I_{t}\right)=\mathbb{E}\left(\hat{\theta}_{t} I_{t}\right) \\
=\mathbb{E}\left(\int_{0}^{t} \Phi(t, s) d \xi_{s} \int_{0}^{t} f(t, s) d \xi_{s}\right)
\end{gathered}
$$




$$
\begin{gathered}
=\int_{0}^{t} \Phi(t, s) f(t, s) d s+\int_{0}^{t} \int_{0}^{t} \Phi(t, \tau) f(t, s) \frac{\partial^{2}}{\partial s \partial r} \Gamma^{h}(\tau, s) d \tau d s \\
+\int_{0}^{t} \int_{0}^{t} \Phi(t, \tau) f(t, s) A(\tau) A(s) K(\tau, s) d \tau d s \\
+\int_{0}^{t} \int_{0}^{t} \Phi(t, \tau) f(t, s) A(s) \frac{\partial \widetilde{K}}{\partial \tau}(s, \tau) d \tau d s \\
+\int_{0}^{t} \int_{0}^{t} \Phi(t, \tau) f(t, s) A(\tau) \frac{\partial \widetilde{K}}{\partial s}(\tau, s) d \tau d s
\end{gathered}
$$

and

$$
\mathbb{E}\left(\theta_{t} \int_{0}^{t} f(t, s) d \xi_{s}\right)=\int_{0}^{t} f(t, s) \frac{\partial \tilde{K}}{\partial s}(t, s) d s+\int_{0}^{t} f(t, s) A(s) K(t, s) d s
$$

Now, $f$ is otherwise arbitrary and the function $\frac{\partial^{2}}{\partial t \partial s} \Gamma^{h}(t, s)=h(2 h-1)|t-s|^{2 h-2}$, so

$$
\int_{0}^{T} \int_{0}^{T}\left(\frac{\partial^{2}}{\partial t \partial s} \Gamma^{h}(t, s)\right)^{2} d t d s<\infty
$$

which means the process $W_{t}+B_{t}^{h}$ is equivalent in an innovation sense [8] to $W_{t}$. Hence equation (5) is valid. Uniqueness follows from the linearity of the equations under consideration.

This completes the proof of Theorem 2.1.

\section{Example}

Consider the case $a(t) \equiv 0$ and $A(t) \equiv 0$, so system (1) reduces to

$$
\theta_{t}=B_{t}^{h}, \quad \xi_{t}=W_{t}+B_{t}^{h}
$$

By Theorem 2.1, the filtering estimate $\widehat{\theta}_{t}=\int_{0}^{t} \Phi(t, s) d \xi_{s}$ and $\Phi$ satisfies the integral equation

$$
\begin{aligned}
\Phi(t, s) & =-\int_{0}^{t} h(2 h-1) \Phi(t \tau)|s-\tau|^{2 h-2} d \tau \\
& +\frac{1}{2} \frac{\partial}{\partial s}\left\{t^{2 h}+s^{2 h}-|t-s|^{2 h}\right\},
\end{aligned}
$$

which is a Fredholm integral equation with a singular kernel. However, for $h>3 / 4$ this kernel is square integrable and equation (11) has a unique solution. In this case, 
the filtering error $\gamma_{t}=\mathbb{E}\left(\left|\theta_{t}-\widehat{\theta}_{t}\right|^{2}\right)$ is given by

$$
\begin{gathered}
\gamma_{t}=\mathbb{E}\left(\left|\theta_{t}\right|^{2}\right)-\mathbb{E}\left(\left|\hat{\theta}_{t}\right|^{2}\right) \\
=t^{2 h}-\mathbb{E}\left(\left|\int_{0}^{t} \Phi(t, s) d \xi_{s}\right|^{2}\right) \\
=t^{2 h}-\int_{0}^{t} \Phi(t, s)\left[\Phi(t, s)+\int_{0}^{t} h(2 h-1) \Phi(t, \tau)|s-\tau|^{2 h-2} d \tau\right] d s \\
=t^{2 h}-\frac{1}{2} \int_{0}^{t} \Phi(t, s) \frac{\partial}{\partial s}\left[t^{2 h}+s^{2 h}-|t-s|^{2 h}\right] d s
\end{gathered}
$$

using (11).

\section{Acknowledgement}

Partially supported by the Australian Research Council Grants A89601825 and C1960019. The authors wish to thank the referee for his many constructive comments.

\section{References}

[1] Dai, Long-range Dependent Processes and Fractional Brownian Motion, Ph.D. thesis, Australian National University 1996.

[2] Kleptsyna, M.L., Kloeden, P.E. and Anh, V.V., Linear filtering with fractional Brownian motion, Stoch. Anal. Appl. (to appear).

[3] Kleptsyna, M.L., Kloeden, P.E. and Anh, V.V., Nonlinear filtering with fractional Brownian motion, J. Info. Trans. Problems (to appear).

[4] Lin, S.J., Stochastic analysis of fractional Brownian motion, Stoch. and Stochastics Rep. 55 (1995), 121-140.

[5] Lipster, R.S. and Shiryayev, A.N., Statistics of Random Processes, Volume II, Springer-Verlag, Berlin 1978.

[6] Lipster, R.S. and Shiryayev, A.N., Theory of Martingales, Springer-Verlag, Berlin 1984.

[7] Mandelbrot, B.B. and Van Ness, J.W., Fractional Brownian motion, fractional noises and applications, SIAM Review 10 (1968), 422-437.

[8] Rozovskii, B.L., Evolutionary Stochastic Systems, Linear Theory with Applications to the Statistics of Random Processes, Nauka, Moscow 1983.

[9] Samorodnitsky, G. and Taqqu, M..S., Stable Non-Gaussian Random Processes, Chapman and Hall, New York 1994. 


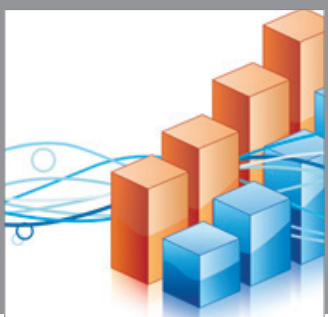

Advances in

Operations Research

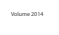

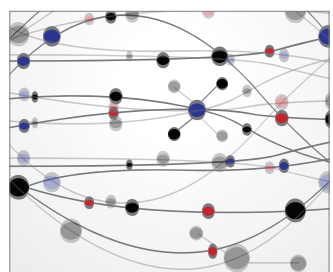

\section{The Scientific} World Journal
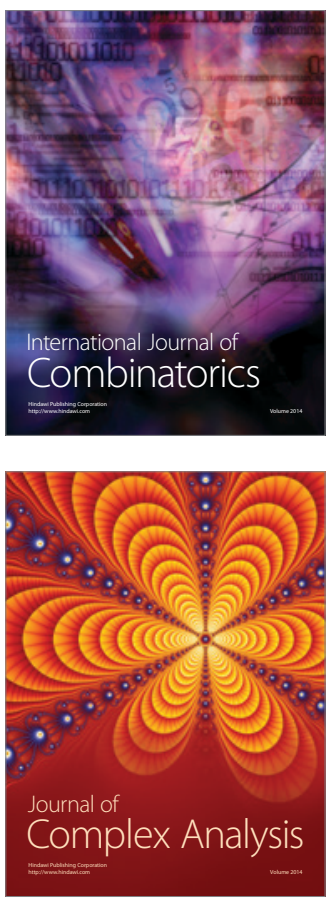

International Journal of

Mathematics and

Mathematical

Sciences
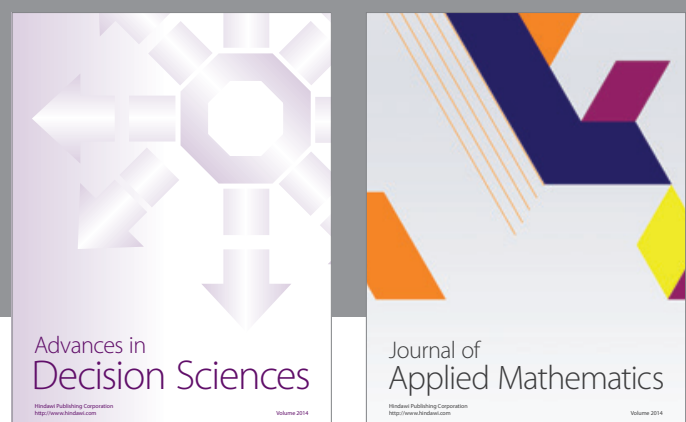

Journal of

Applied Mathematics
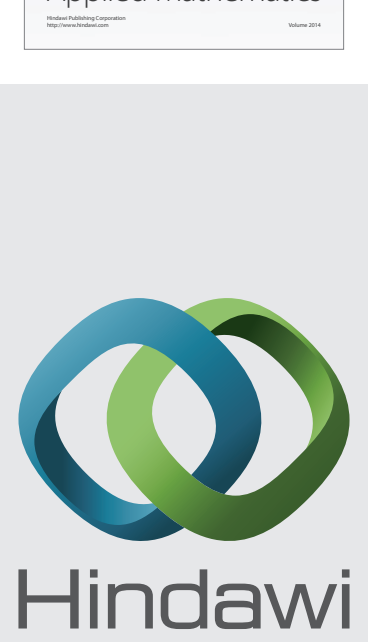

Submit your manuscripts at http://www.hindawi.com
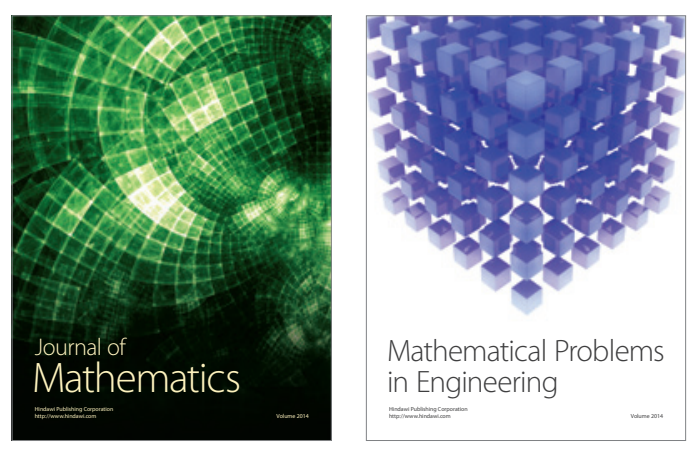

Mathematical Problems in Engineering
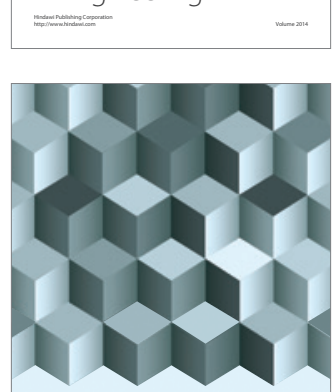

Journal of

Function Spaces
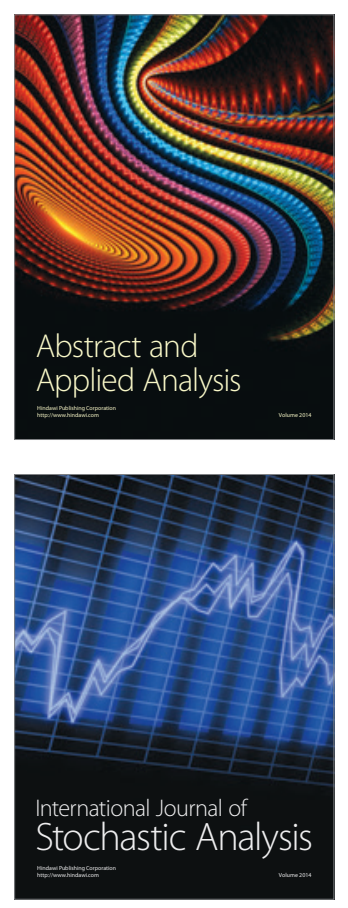

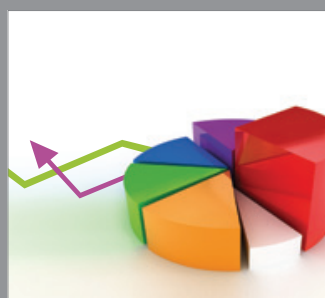

ournal of

Probability and Statistics

Promensencen
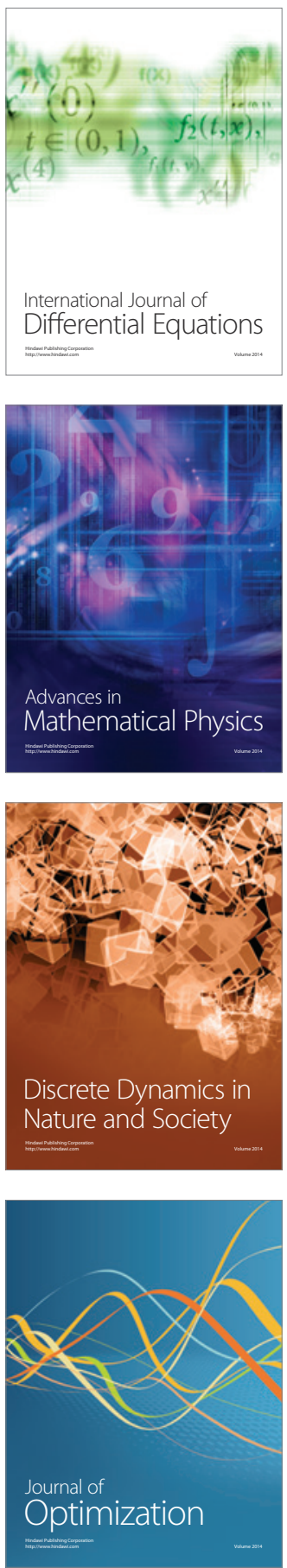